\title{
Disease-Specific Electrocardiographic Lead Positioning for Early Detection of Arrhythmogenic Right Ventricular Cardiomyopathy
}

\author{
Janna Ruisch ${ }^{1,2}$, Machteld J Boonstra ${ }^{2}$, Rob W Roudijk ${ }^{2}$, Peter M van Dam² ${ }^{2}$ Cornelis H Slump ${ }^{1}$, \\ Peter Loh $^{2}$ \\ ${ }^{1}$ University of Twente, Enschede, The Netherlands \\ ${ }^{2}$ University Medical Center Utrecht, Utrecht, The Netherlands
}

\begin{abstract}
Arrhythmogenic right ventricular cardiomyopathy $(A R V C)$ is characterized by replacement of cardiomyocytes by fibrofatty tissue which can lead to ventricular arrhythmias, heart failure or sudden cardiac death. Genetic defects in desmosomal proteins, as plakophilin-2 (PKP2), are known to contribute to disease development. Current electrocardiographic (ECG) criteria for ARVC diagnosis only focus on right precordial leads, but sensitivity of current depolarization criteria is limited. This study aimed to identify additional depolarization criteria with most optimal lead configurations for early detection of ARVC in PKP2 pathogenic mutation carriers. In PKP2-positive ARVC patients (n=7), PKP2 pathogenic variant carriers $(n=16)$ and control subjects without structural heart disease $(n=9), 67-l e a d$ body surface potential maps (BSPM) were obtained. Terminal QRS-integrals were determined and quantitatively compared to controls using departure mapping. Significantly different terminal QRS-integrals were identified in lead 34 (conventional V3), 40 and 41 (conventional V4). To conclude, a clear distinction between ARVC patients, asymptomatic mutation carriers and healthy controls was observed.
\end{abstract}

\section{Introduction}

Arrhythmogenic right ventricular cardiomyopathy (ARVC) is characterized by replacement of cardiomyocytes by fibrofatty tissue that may lead to sudden cardiac death, ventricular arrhythmias or heart failure. Genetic defects in desmosomal proteins, with plakophilin-2 (PKP2) being the most frequently affected gene, contribute to disease development. The current 2010 Task Force Criteria (TFC) for diagnosis of ARVC are based on a set of major and minor criteria from different diagnostic modalities. However, diagnosis of ARVC is complex due to a heterogenous clinical presentation of the disease. ${ }^{1-3}$
The association between structural and electrical progression in ARVC patients has been demonstrated. ${ }^{4}$ Significant structural right ventricular (RV) progression were associated with prior depolarization abnormalities. Therefore, accurate detection of electrical abnormalities is of utmost importance for early detection of ARVC. Current electrocardiographic (ECG) criteria of ARVC focus on the presence of abnormalities (e.g. epsilon waves, prolonged terminal activation duration (TAD) or negative T-waves) in right precordial leads V1-V3. However, only negative $\mathrm{T}$-waves showed to be highly sensitive (79\%) compared to prolonged TAD (40\%) and epsilon waves (10\%). ${ }^{1,5}$

The amount of information obtained with conventional 12-lead ECG suffices for most clinical applications. However, in some applications the use of extra ECG leads or other lead locations has proven to increase the detection rate, like Brugada Syndrome or posterior or inferior myocardial infarction. ${ }^{6-8}$ This study aims to determine additional depolarization criteria and optimal lead positioning for early detection of ARVC in PKP2 pathogenic variant carriers.

\section{Method}

\subsection{Study population}

The study population consisted of PKP2 pathogenic variant carriers $(n=23)$ and control subjects $(n=9)$ with symptomatic ventricular extrasystoles originating in the right ventricular outflow tract (RVOT VES). Exclusion criteria were a right bundle branch block (QRS width > $120 \mathrm{~ms}$ ) and other structural diseases that affect ECG morphology apart from ARVC symptoms. Besides, controls that showed signs of heart failure were excluded in the study (LV or RV ejection fraction $<45 \%, \mathrm{RV}$ enddiastolic volume index $>110 \mathrm{ml} / \mathrm{m}^{2}$ ). PKP2 pathogenic variant carriers were tested to meet the TFC for ARVC and assigned to either the ARVC subgroup (TFC $\geq 4$ ) or PKP2 subgroup. Informed consent was obtained from all subjects. The study was approved by the Medical Ethics Committee of University Medical Center Utrecht (17/907). 

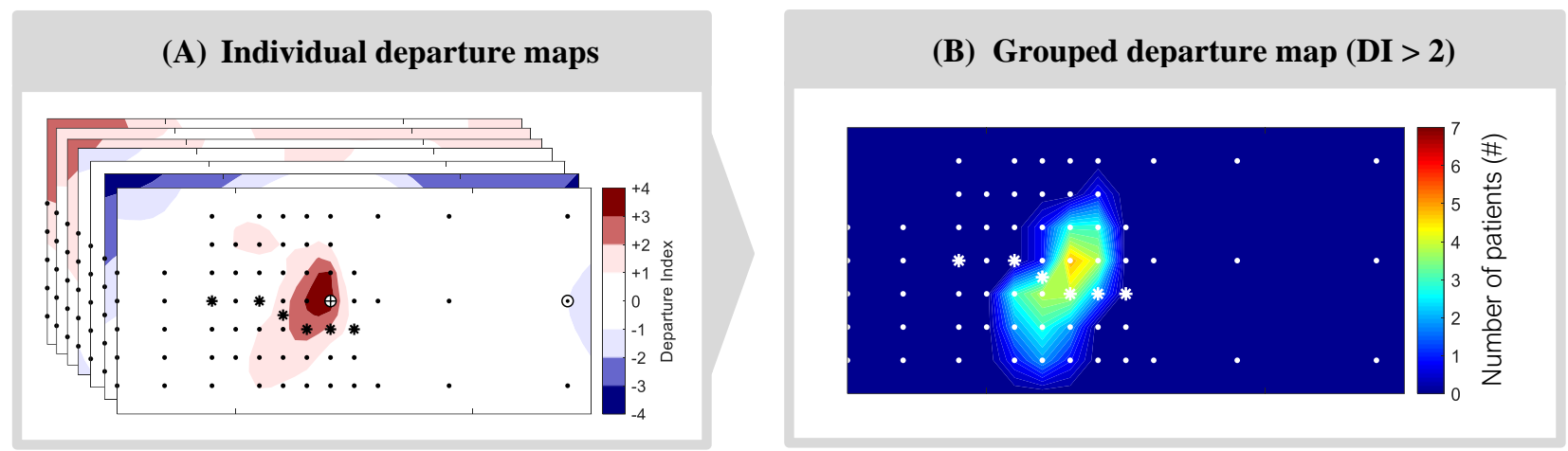

Figure 1. Overview that visualizes the combining process of all individual departure maps toward one single grouped departure map. (A) Seven individual departure maps (ARVC vs CONTROL) with the departure index (DI) per lead position. (B) Grouped departure map, where the value at each lead corresponds to the total ARVC subjects that show significantly different integrals (DI > 2) in the body surface potential map. The small dots indicate all BSPM electrodes and the stars refer to the conventional 12-lead electrode positions.

\subsection{Body surface potential mapping}

67-lead body surface potential map (BSPM) were obtained in each subject, consisting of 64 precordial and 3 limb leads (Figure 1, small dots). The anterior body surface includes 55 leads, that were placed in nine vertical strips. Within the vertical strips, leads were separated $4 \mathrm{~cm}$ from each other. The posterior body surface includes 9 leads, that were placed in three vertical columns. BSPM were recorded (sampling frequency $2048 \mathrm{~Hz}, 24 \mathrm{bits} / \mathrm{sample}$, Biosemi, Amsterdam, The Netherlands). Precordial leads were referenced to Wilson's central terminal. BSPMs were down-sampled to $1000 \mathrm{~Hz}$. Leads with noise were removed.

\subsection{Data analysis}

Per subject, ten consecutive sinus beats obtained during resting respiration were averaged. QRS-onset and QRSend were manually annotated using the root mean square (RMS) of all leads. Early electrical depolarization abnormalities of ARVC disease in PKP2 pathogenic variant carriers were investigated by comparing integral maps from BSPM data. Integral maps were computed of the last $60 \mathrm{~ms}$ of the QRS complex (terminal QRS integral), which indicates a mean potential direction over this interval.

\subsection{Departure mapping technique}

The departure index (DI) was used to determine the extent of integral deviation from the normal range in the control group. The DI was calculated by:

$$
D I=\frac{I_{N}-\mu_{\text {control }}}{\sigma_{\text {control }}}
$$

where $I_{N}$ equals the integral of an individual subject $N$, $\mu_{\text {control }}$ is the mean integral of control group and $\sigma_{\text {control }}$ is the standard deviation of the integrals in control group. Both ARVC subjects and PKP2 pathogenic variant carriers were compared with the control group. DI was calculated for each integral per lead and integrals significantly differing (> 2 SD) from the control data were classified as abnormal. Per subgroup, individual departure maps were summed to identify significant differences per group (Figure 1).

Receiver Operating Characteristic (ROC) curves were used to define optimal cut-off values for ARVC classification. The diagnostic values of ARVC of additional ECG parameters and lead positions were determined by calculating the sensitivity (SE) and specificity (SP).

\section{Results}

From all PKP2 pathogenic variant carriers, seven subjects met the TFC for ARVC. Patient characteristics are stated in Table 1.

Table 1: Patient characteristics

\begin{tabular}{lccc}
\hline Variables & $\begin{array}{c}\text { ARVC } \\
(\mathrm{n}=7)\end{array}$ & $\begin{array}{c}\text { PKP2 } \\
(\mathrm{n}=16)\end{array}$ & $\begin{array}{c}\text { CONTROL } \\
(\mathrm{n}=9)\end{array}$ \\
\hline Male & 4 & 5 & 3 \\
Age $(\mathrm{y})$ & $36 \pm 18$ & $38 \pm 17$ & $34 \pm 13$ \\
TFC $(\#)$ & $6.6 \pm 2.4$ & $2.2 \pm 0.4$ & - \\
Onset diagnosis $(\mathrm{y})$ & $3.7 \pm 3.8$ & - & - \\
QRS width (ms) & $103 \pm 8$ & $106 \pm 6$ & $105 \pm 9$ \\
Heart rate $(\mathrm{bpm})$ & $61 \pm 10$ & $61 \pm 11$ & $63 \pm 9$ \\
LV EDVI $\left(\mathrm{ml} / \mathrm{m}^{2}\right)$ & $93 \pm 15$ & $94 \pm 11$ & $95 \pm 17$ \\
LV EF $(\%)$ & $59 \pm 5$ & $55 \pm 4$ & $54 \pm 5$ \\
RV EDVI $\left(\mathrm{ml} / \mathrm{m}^{2}\right)$ & $129 \pm 40$ & $92 \pm 13$ & $92 \pm 14$ \\
RV EF $(\%)$ & $41 \pm 13$ & $56 \pm 6$ & $53 \pm 6$ \\
\hline AbbeV
\end{tabular}

Abbreviations: $\mathrm{LV}=$ left ventricle. $\mathrm{EDVI}=$ end-diastolic volume index. $\mathrm{EF}=$ ejection fraction. $\mathrm{RV}=$ right ventricle. $\mathrm{TFC}=$ Task Force Criteria. 


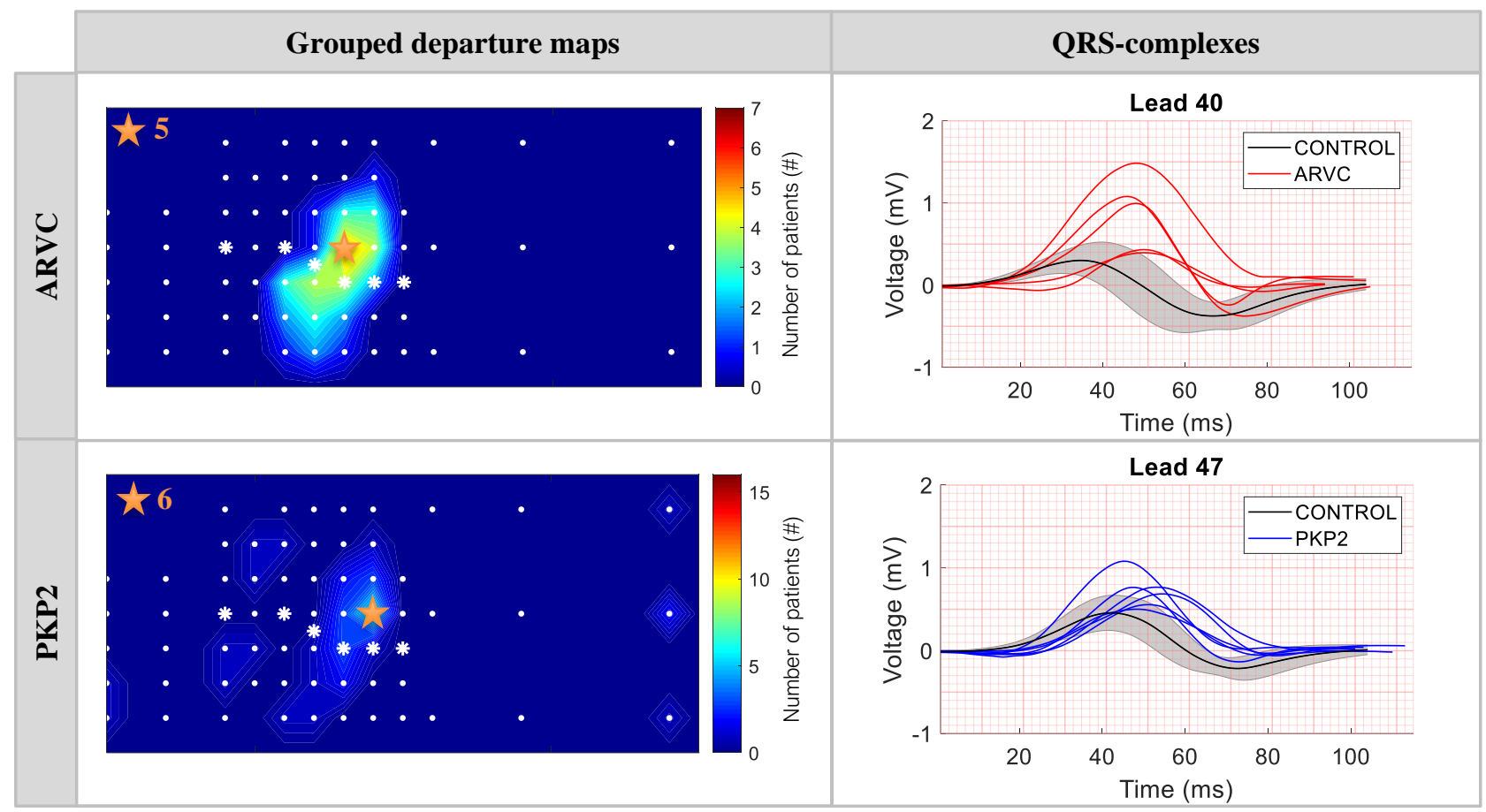

Figure 2. Grouped departure maps (DI > 2) of terminal QRS-integrals (left) and QRS-complexes of orange stared lead positions (right). The grouped departure maps and QRS-potentials of ARVC vs CONTROL (upper row) and PKP2 vs CONTROL (bottom row) were visualized. Orange stars correspond to leads where most subjects showed significantly different integrals. Maximum was noted in left-upper corner of the grouped departure map.

Lead 40 is significantly different in five out of seven ARVC patients (Figure 2, orange star). Six out of 16 PKP2 pathogenic variant carriers showed different terminal QRS-integrals in lead 47. The QRS-complexes of five ARVC subjects in lead 40 and six PKP2 pathogenic variant carriers in lead 47 were visualized in Figure 2.

The difference in both terminal QRS-integrals and Rwave apex duration were determined in conventional 12lead positions, lead 40 and 47 (Table 2) for both groups.

\section{Discussion}

The purpose of this study was to identify additional depolarization parameters and optimal lead placement for the early detection of ARVC in PKP2 pathogenic variant carriers. Significant differences were found in terminal QRS-integrals compared to the control group. The results indicate that depolarization abnormalities can be observed in other leads beyond conventional 12-lead ECG positions V1-V3. From the grouped departure map, lead 40 (above lead 41 which corresponds to conventional V4) showed significant increased terminal QRS-integrals (DI >2) in five out of seven ARVC patients. In PKP2 pathogenic variant carriers, lead 47 (above lead 48 which position corresponds to conventional V5) showed significantly different integrals.

Significantly higher terminal QRS-integrals in both ARVC and PKP2 pathogenic variant carriers, can be explained by increased R-wave amplitudes $(\mathrm{mV})$, delayed $\mathrm{R}$-wave apex (ms), and decreased S-wave amplitude ( $\mathrm{mV}$ ) in QRS-complexes (Figure 2). These changes might be declared by defects in cell-cell adhesion, caused by the presence of PKP2 pathogenic gene variants in desmosomal proteins. Desmosomes are essential to withstand mechanical stresses caused by the contractile function of the heart. Segments with largest mechanical stress during the cardiac cycle were most commonly structural affected in ARVC, like the inflow tract, outflow tract or apex of the RV. This study observed electrical changes in ARVC patients are not only present in the RV, but can appear in $\mathrm{LV}$. PKP2 is known to be a right ventricular dominant disease, but based on results in this study the right-sided phenotype might only be valid for structural abnormalities. This hypothesizes that the area of electrical abnormalities does not have to agree with the area of structural abnormalities, which substantiates the need of revision of the current ECG TFC of ARVC.

Despite the known heterogenous clinical presentation of ARVC, terminal QRS-integrals and R-wave apex duration showed to be a highly sensitive (>86\%) and specific $(>89 \%)$ criteria in lead V3, V4 and 40 for all subjects. Using these cut-off values in PKP2 pathogenic variant carriers, up to $44 \%$ were classified with electrical abnormalities that were found in ARVC patients.

This study excluded patients with prolonged QRSduration (QRS > 120ms) to focus on initial depolarization 
Table 2. Differentiation of ARVC subjects $(n=7)$ and PKP2 pathogenic variant carriers $(n=16)$ from control subjects $(n=9)$ for both conventional 12-lead ECG positions and new lead positions found with the departure mapping technique.

\begin{tabular}{|c|c|c|c|c|c|c|c|c|c|c|}
\hline \multirow[b]{3}{*}{ Lead } & \multicolumn{5}{|c|}{ Terminal QRS-integral (mVms) } & \multicolumn{5}{|c|}{ R-wave apex duration (ms) } \\
\hline & \multirow{2}{*}{$\begin{array}{c}\mathrm{CO} \\
(\mathrm{mVms})\end{array}$} & \multicolumn{2}{|c|}{ ARVC } & \multicolumn{2}{|c|}{ PKP2 } & \multirow{2}{*}{$\begin{array}{c}\mathrm{CO} \\
(\mathrm{ms})\end{array}$} & \multicolumn{2}{|c|}{ ARVC } & \multicolumn{2}{|c|}{ PKP2 } \\
\hline & & SE $(\%)$ & $\mathrm{SP}(\%)$ & SE $(\%)$ & $\mathrm{SP}(\%)$ & & $\mathrm{SE}(\%)$ & $\mathrm{SP}(\%)$ & SE $(\%)$ & SP $(\%)$ \\
\hline 14 (V1) & -2.9 & 29 & 100 & 6 & 100 & 10 & 71 & 78 & 25 & 78 \\
\hline $26(\mathrm{~V} 2)$ & -13.9 & 57 & 89 & 13 & 89 & 15 & 86 & 78 & 56 & 78 \\
\hline *33-34 (V3) & -11.9 & 100 & 89 & 44 & 89 & 28 & 86 & 89 & 25 & 89 \\
\hline 41 (V4) & -1.7 & 100 & 89 & 44 & 89 & 37 & 86 & 100 & 25 & 100 \\
\hline 48 (V5) & 16.6 & 57 & 89 & 13 & 89 & 37 & 86 & 67 & 50 & 67 \\
\hline $53(\mathrm{~V} 6)$ & 18.2 & 43 & 78 & 13 & 78 & 38 & 57 & 67 & 50 & 67 \\
\hline 40 & -1.9 & 100 & 100 & 31 & 100 & 32 & 100 & 89 & 38 & 89 \\
\hline 47 & 4.3 & 86 & 89 & 38 & 89 & 40 & 43 & 89 & 19 & 89 \\
\hline
\end{tabular}

Abbreviations: $\mathrm{CO}=$ cut-off value. $\mathrm{SE}=$ sensitivity. $\mathrm{SP}=$ specificity.

* The average of lead 33 and 34 corresponds with conventional V3 position.

abnormalities as we assumed that first electrical changes caused by disease progression do not directly result in increased QRS-duration. Besides, RVOT VES patients were used as controls. A future clinical validation has to be performed in healthy subjects without RVOT VES, as due to the occurrence of RVOT VES the absence of an underlying pathological process cannot be guaranteed. Electrical abnormalities in RVOT VES subjects might affect the results found with the departure mapping technique. Furthermore, a clinical validation of these additional parameters is required to relate electrical changes with early disease progression. Alterations of electrical abnormalities that were also observed in PKP2 pathogenic variant carriers require longer follow-up to relate electrical abnormalities to early disease progression of ARVC.

\section{Conclusion}

This study revealed additional lead positions beside conventional 12-lead ECG positions in which electrical changes were observed that may be used to identify the onset of disease in pathogenic mutation carriers. Similar depolarization changes in both ARVC patients and PKP2 pathogenic variant carriers were shown. The additional lead positions in combination with the newly identified depolarization criteria, are likely to improve detection of the early onset of disease in PKP2 pathogenic variant carriers.

\section{Acknowledgments}

This work was supported by the Netherlands Cardiovascular Research Initiative, an initiative with support of the Dutch Heart Foundation (grant numbers CVON2015-12 eDETECT and QRS-Vision 2018B007).

\section{References}

[1] Marcus FI. Diagnosis of ARVC; proposed modification of the task force criteria. Eur Heart J. 2010;31(7):1533-1541.

[2] Quarta G, Elliott PM. Diagnostic criteria for arrhythmogenic right ventricular cardiomyopathy. Rev Esp Cardiol. 2012;65(7):599-605.

[3] Bosman LP, Verstraelen TE, van Lint FHM, et al. The Netherlands Arrhythmogenic Cardiomyopathy Registry: design and status update. Neth Heart J. 2019;27(10):480486.

[4] Mast TP, James CA, Calkins H, et al. Evaluation of structural progression in arrhythmogenic right ventricular dysplasia / cardiomyopathy. JAMA Cardiol. 2017;2(3):293-302.

[5] Bhonsale A, te Riele ASJM, Sawant AC, et al. Cardiac phenotype and long-term prognosis of arrhythmogenic right ventricular cardiomyopathy/dysplasia patients with late presentation. Heart Rhythm. 2017;14(6):883-891.

[6] Priori S, Blomstro C, Blom N, et al. 2015 ESC Guidelines for the management of patients with ventricular arrhythmias and the prevention of sudden cardiac death. Eur Soc Cardiol. 2015;17:1601-1687.

[7] Shimizu W, Takagi M, Aiba T, Taguchi A, Kurita T, Kamakura S. Body surface distribution and response to drugs of ST-segment elevation in Brugada Syndrome: clinical implication of eighty-seven-lead body surface potential mapping and its application to twelve-lead electrocardiograms. $J$ Cardiovasc Electrophysiol. 2000;11(4):396-404.

[8] Ibanez B, James S, Agewall S, et al. 2017 ESC Guidelines for the management of acute myocardial infarction in patients presenting with ST-segment elevation. Eur Heart J. 2018;39(2):119-177.

Address for correspondence:

Janna Ruisch

Heidelberglaan 100, 3584 CX, Utrecht, The Netherlands

Email: j.ruisch@umcutrecht.nl 